\title{
Substrate-emitting ring interband cascade lasers
}

Martin Holzbauer, Rolf Szedlak, Hermann Detz, Robert Weih, Sven Höfling, Werner Schrenk, Johannes Koeth, and Gottfried Strasser

Citation: Appl. Phys. Lett. 111, 171101 (2017);

View online: https://doi.org/10.1063/1.4989514

View Table of Contents: http://aip.scitation.org/toc/apl/111/17

Published by the American Institute of Physics

\section{Scilight}

Sharp, quick summaries illuminating the latest physics research 


\title{
Substrate-emitting ring interband cascade lasers
}

\author{
Martin Holzbauer, ${ }^{1, a)}$ Rolf Szedlak, ${ }^{1}$ Hermann Detz, ${ }^{1,2}$ Robert Weih,, ${ }^{3,4}$ Sven Höfling, ${ }^{3,5}$ \\ Werner Schrenk, ${ }^{1}$ Johannes Koeth, ${ }^{4}$ and Gottfried Strasser ${ }^{1}$ \\ ${ }_{1}^{1}$ Institute of Solid State Electronics and Center for Micro- and Nanostructures, TU Wien, 1040 Vienna, Austria \\ ${ }^{2}$ Austrian Academy of Sciences, 1010 Vienna, Austria \\ ${ }^{3}$ Technische Physik, Physikalisches Institut and Wilhelm-Conrad-Röntgen Research Center for Complex \\ Material Systems, University of Würzburg, 97074 Würzburg, Germany \\ ${ }^{4}$ nanoplus Nanosystems and Technologies GmbH, 97218 Gerbrunn, Germany \\ ${ }^{5}$ SUPA, School of Physics and Astronomy, University of St Andrews, St Andrews KY16 9SS, United Kingdom
}

(Received 9 June 2017; accepted 15 October 2017; published online 26 October 2017)

\begin{abstract}
We demonstrate interband cascade lasers fabricated into ring-shaped cavities with vertical light emission through the substrate at a wavelength of $\lambda \approx 3.7 \mu \mathrm{m}$. The out-coupling mechanism is based on a metallized second-order distributed feedback grating. At room-temperature, a pulsed threshold current-density of $0.75 \mathrm{kA} / \mathrm{cm}^{2}$ and a temperature-tuning rate of $0.3 \mathrm{~nm} /{ }^{\circ} \mathrm{C}$ are measured. In contrast to the azimuthal polarization of ring quantum cascade lasers, we observe a radial polarization of the projected nearfield of ring interband cascade lasers. These findings underline the fundamental physical difference between light generation in interband and intersubband cascade lasers, offering new perspectives for device integration. (C) 2017 Author(s). All article content, except where otherwise noted, is licensed under a Creative Commons Attribution (CC BY) license (http://creativecommons.org/licenses/by/4.0/). https://doi.org/10.1063/1.4989514
\end{abstract}

The mid-infrared spectral region $(3-10 \mu \mathrm{m})$ is of great interest for spectroscopic applications due to strong vibrational and rotational molecular absorption features. The demand for compact, portable, and energy-efficient laser sources for selective measurements of trace gases leads to the development of different device concepts. While interband laser diodes ${ }^{1}$ dominate in the lower wavelength region up to $3.3 \mu \mathrm{m}$, the quantum cascade laser $^{2}$ (QCL) has become a well-established laser source from $3 \mu \mathrm{m}$ up to the $\mathrm{THz}$ spectral range. Laser diodes are based on interband transitions between states in the conduction and valence bands with typically long lifetimes (in the order of ns) in the excited states. In contrast to that, QCLs are unipolar devices that rely on intersubband transitions within the conduction band, where lifetimes are typically three orders of magnitude shorter. ${ }^{3}$ To compensate for the lower gain, a serial cascading of stages is introduced.

The interband cascade laser ${ }^{4,5}$ (ICL) combines the conduction-to-valence band transitions in laser diodes with the voltage-efficient cascading in QCLs. Although ICLs may have higher internal losses compared to QCLs, the differential gain per unit current density per stage exceeds the latter ones by two orders of magnitude. ${ }^{3}$ Rebalancing of the internal electron and hole densities has been identified as a crucial design task to improve the performance of ICLs. ${ }^{6} \mathrm{~A}$ heavy n-doping of the electron injector is used to compensate the far higher hole densities, leading to a lower threshold power consumption. State-of-the-art GaSb-based ICLs can emit light at room temperature in the 2.8 to $5.6 \mu \mathrm{m}$ wavelength region. ${ }^{6-9}$

To achieve single-mode operation, a wavelength selection mechanism is necessary. A common way is to incorporate a distributed-feedback (DFB) grating into the laser

a)Electronic mail: martin.holzbauer@tuwien.ac.at waveguide. Different grating concepts have been already demonstrated for facet emitting ridge lasers, e.g., corrugated sidewalls, ${ }^{10}$ germanium top-gratings, ${ }^{11}$ or lateral metal gratings. ${ }^{12}$ To extend the current-limited spectral tuning bandwidth of DFB lasers from a few up to hundreds of nanometers, e.g., a two-segment ICL device together with binary superimposed gratings ${ }^{13}$ can be used.

However, since these devices rely on edge-emission from a cleaved facet, a full process run is necessary to verify the proper operation of the laser. For quality monitoring, an in-production on-chip testing capability would be beneficial. Furthermore, the position of the cleave edge with respect to the grating does have a strong impact on the device performance. Alternative solutions are laser devices that emit light through the surface layers, rather than via a cleave edge. In addition, surface emission enables two-dimensional array integration of multiple devices. Recently, an electrically pumped interband cascade vertical-cavity surface-emitting laser (VCSEL) has been demonstrated ${ }^{14}$ in pulsed operation at a wavelength of $\lambda \approx 3.4 \mu \mathrm{m}$. Another VCSEL device ${ }^{15}$ that is based on a single stage active region design $(\lambda=4 \mu \mathrm{m})$ operated in continuous-wave up to $-7^{\circ} \mathrm{C}$ and in pulsed mode up to $45^{\circ} \mathrm{C}$. For these devices, the feedback mechanism is provided by high-quality Bragg mirrors grown below and above the ICL gain material.

In this work, we present another approach to achieve vertical light emission ${ }^{16,17}$ from interband cascade lasers, which is based on a 2nd-order DFB grating. Herein, the first-order diffraction of the guided modes enables vertical light emission. We combine the DFB grating with a ring-shaped ${ }^{18-20}$ laser waveguide, which creates a circular symmetric beam pattern and allows a better collimation of the emitted light due to the larger aperture. ${ }^{21}$ In ring waveguides, two counterpropagating whispering gallery modes are coupled by the periodic modulation of the DFB grating. In particular, the 
solutions of the infinite-length grating-waveguide problem are characterized by symmetric (radiating) and antisymmetric (non-radiating) modes. ${ }^{22}$ Since the losses for the antisymmetric mode are in general lower, this mode is favored over the symmetric one. Therefore, the radiating mode would not reach the lasing threshold and light emission is prohibited. However, the losses for the non-radiating mode can be increased by, e.g., coupling to a metal. ${ }^{23}$ In this case, the losses are then lower for the symmetric mode and vertical light emission via the surface is possible.

In this study, a 2nd-order DFB grating is etched into the upper InAs/AlSb superlattice cladding of an ICL gain material, where the surface and the grooves are subsequently covered with metal. The device is designed for light emission through the GaSb substrate. As the area on the substrate-side can still be used to integrate other optical elements, such as lenses ${ }^{24}$ or polarizers, ${ }^{25}$ compact sensing systems with more functionalities are possible.

We calculate the coupling coefficient of the 2nd-order DFB metallized grating within the framework of the coupled-mode theory. Hereby, we search for Floquet-Bloch solutions of the infinite-length grating-waveguide problem ${ }^{26}$ adopted to TE-polarized laser structures. This theory has been developed especially for the case of "strong" metallized gratings, where the original approach of Kogelnik and Shank $^{27}$ is not applicable anymore. The important design parameters are the grating etch depth and the grating dutycycle $\sigma$, where the latter we define as the ratio between etched grooves and grating period $\Lambda_{\mathrm{g}}$. In other words, $\sigma$ corresponds to the amount of metal per grating period. For the fabricated DFB grating, we estimated a coupling strength of $\left|\kappa L_{\text {ring }}\right| \sim 1$, where $\kappa$ is the coupling coefficient and $L_{\text {ring }}=1.23 \mathrm{~mm}$ the length of the ring ICL device.

The ICL structure is based on a 6-stage active region design, which is grown by molecular beam epitaxy on a single-side polished $2 \times 10^{18} \mathrm{~cm}^{-3} \mathrm{n}$-doped GaSb (100) substrate. The active region is sandwiched between two $200 \mathrm{~nm}$ thick GaSb separate confinement layers to concentrate the optical mode in the centre of the waveguide and hence ensure a high modal gain. Furthermore, the material stack is surrounded by InAs/AlSb superlattice cladding layers. The fabrication of the ring lasers starts with the deposition of a $\mathrm{Si}_{x} \mathrm{~N}_{y}$ hardmask via plasma enhanced chemical vapor deposition. This mask layer is structured by optical lithography into ring-shaped cavities with an outer diameter of $D=400 \mu \mathrm{m}$ and a waveguide width of $w=10 \mu \mathrm{m}$. An anisotropic reactive ion etching (RIE) process is used to etch the waveguide into the lower cladding layers. Subsequently, the 2nd-order DFB grating is written by e-beam lithography with a grating period of $\Lambda_{\mathrm{g}}=1.104 \mu \mathrm{m}$ and a grating duty-cycle of $\sigma=59 \%$ (measured in the middle of the waveguide). Using a $\mathrm{Si}_{x} \mathrm{~N}_{y}$ hard mask, the grating is etched $1.1 \mu \mathrm{m}$ into the upper waveguide layers with an anisotropic RIE process yielding vertical grating grooves. A $450 \mathrm{~nm}$ thick $\mathrm{Si}_{x} \mathrm{~N}_{y}$ passivation layer is deposited and selectively removed (opening window width $\sim 9.4 \mu \mathrm{m}$ ) for the top contact metallization. Sputtered Ti/Au layers $(10 \mathrm{~nm} / 500 \mathrm{~nm})$ cover the entire device, including the grating grooves. For the single-side polished $\mathrm{GaSb}$ substrate, a root mean square roughness of $\sim 133 \mathrm{~nm}(30 \mu \mathrm{m} \times 30 \mu \mathrm{m})$ is measured using atomic force microscopy. The substrate is

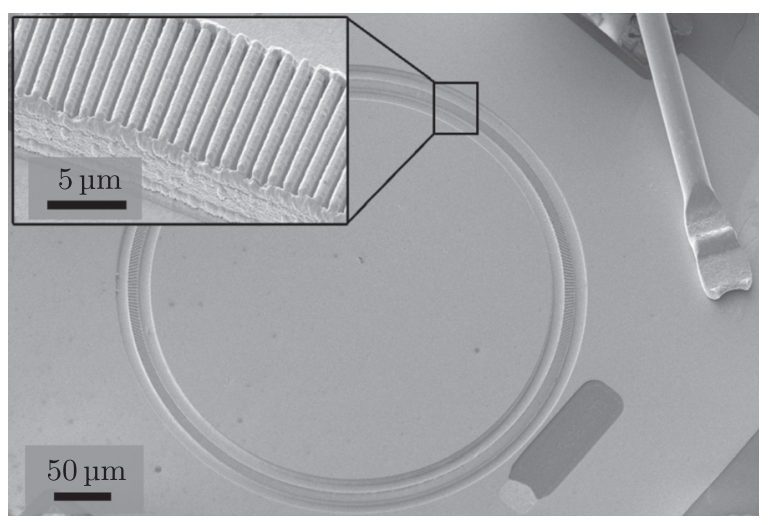

FIG. 1. Scanning electron microscopy image of a ring interband cascade laser. The device has an outer diameter of $D=400 \mu \mathrm{m}$ and the waveguide width is $w=10 \mu \mathrm{m}$. The inset shows a close-up of the metal-covered 2ndorder distributed feedback grating.

neither thinned nor polished. For a target wavelength around $3.7 \mu \mathrm{m}$, we estimated a free carrier absorption coefficient of $\sim 0.015 \mathrm{~cm}^{-1}$, which corresponds to a transmission of $99.9 \%$ through the substrate with a thickness of $\sim 550 \mu \mathrm{m}$. Figure 1 shows a scanning electron microscopy image of the final ring ICL device.

For characterization, the sample is mounted epi-side up using silver paste on a copper plate with an $8 \mathrm{~mm}$ hole for substrate emission. The light is collected from the substrateside with a $\mathrm{ZnSe}$ lens and focused on a wide-range deuterated triglycine sulfate (DTGS) detector of a Bruker Vertex $70 \mathrm{v}$ Fourier transform infrared (FTIR) spectrometer. The ring ICL is operated in pulsed operation at $5 \mathrm{kHz} / 100 \mathrm{~ns}$. Figure 2 shows the light-current-voltage characteristics of the device at different heatsink temperatures $\left(10^{\circ} \mathrm{C}\right.$ to $35^{\circ} \mathrm{C}$ ). For the ring with $400 \mu \mathrm{m}$ diameter and $10 \mu \mathrm{m}$ waveguide width, a pulsed threshold current density of $0.75 \mathrm{kA} / \mathrm{cm}^{2}\left(I_{\mathrm{th}}=92 \mathrm{~mA}\right)$ is measured at a submount temperature of $20^{\circ} \mathrm{C}$. From measurements of the threshold current density $J_{\text {th }}$ as a function of heatsink temperature $T$ and an exponential fit with $J_{\text {th }}(T)=J_{0} \exp \left(T / T_{0}\right)$, a characteristic temperature of $T_{0}=66 \mathrm{~K}$ together with $J_{0}=0.55 \mathrm{kA} / \mathrm{cm}^{2}$ is extracted. The measured threshold current density is comparably high for an ICL, which we attribute to an increase of the absorption losses due to the enhanced overlap of the

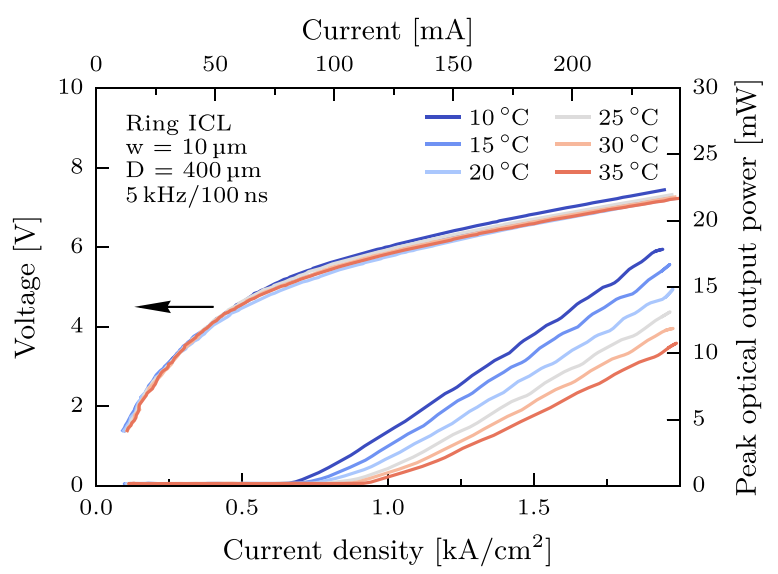

FIG. 2. Light-current-voltage characteristics of a ring ICL device in pulsed operation for different heatsink temperatures. The light is diffracted via a 2nd-order DFB grating towards the substrate at an emission wavelength of $\lambda \approx 3.7 \mu \mathrm{m}$. 
waveguide mode with the metal grating. Unfortunately, continuous wave operation could not be reached due to limited heat dissipation capabilities of the epi-side up mounting of the devices. Figure 3(a) shows a simulation of the first-order whispering gallery mode in the ring ICL. The plot illustrates the time-averaged power flow into the plane of the drawing for the ring-shaped waveguide. In contrast to straight ridges, the fundamental mode is shifted more towards the outer sidewall. Hence, it is more sensitive to surface roughness caused by, e.g., dry-etched sidewalls. The extracted differential slope efficiency for the ring ICL is $104 \mathrm{~mW} / \mathrm{A}$ at $20^{\circ} \mathrm{C}$, which corresponds to an external differential quantum efficiency of $\sim 31 \%$. In comparison, a single-stage VCSEL device $^{15}$ with a buried tunnel junction $(12 \mu \mathrm{m}$ aperture size $)$ showed a maximum slope efficiency of $26 \mathrm{~mW} / \mathrm{A}$ at $-40^{\circ} \mathrm{C}$. A 15 -stage interband cascade VCSEL ${ }^{14}$ with $60 / 40 \mu \mathrm{m}$ mesa/ aperture diameter reached $\sim 52 \mathrm{~mW} / \mathrm{A}$ at $20^{\circ} \mathrm{C}$. Furthermore, the output power of ring ICLs can be scaled by changing the diameter and width of the waveguide. The relatively small gain volume in VCSELs on the one hand limits the maximum output power, but it also enables to build surface-emitting and inherent single-mode devices ${ }^{28}$ without any diffraction grating.

For spectral characterization, the FTIR is operated in the rapid-scan mode and the ring laser is pulsed with $80 \mathrm{kHz} / 30 \mathrm{~ns}$. Figure 4 shows the lasing spectra of the ring ICL device at different heatsink temperatures at $I=130 \mathrm{~mA}$. In the temperature range between 10 and $20^{\circ} \mathrm{C}$, we observe an emission peak at $\lambda=3.73 \mu \mathrm{m}$. For higher temperatures, a second emission peak appears around $\lambda \sim 3.77 \mu \mathrm{m}$. Simulations of the ring waveguide ( $10 \mu \mathrm{m}$ width) confirm that several radial whispering gallery modes with comparable waveguide losses are supported. Figure 3(b) shows the normalized time-averaged power-flow for the first four radial

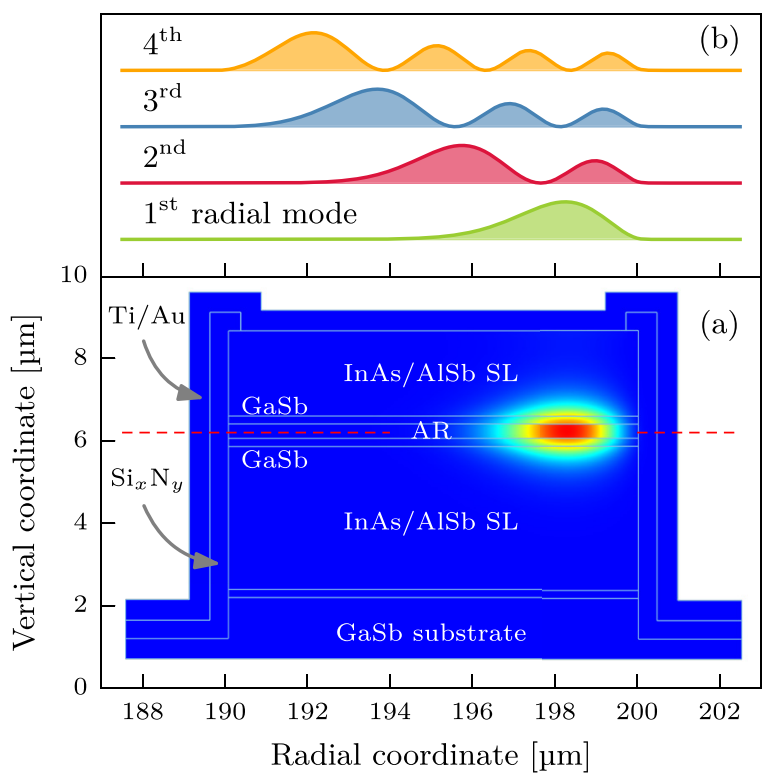

FIG. 3. (a) Simulated mode profile of the 1st-order radial waveguide mode in the ring ICL. The plot shows the time-averaged power-flow. Compared to a straight ridge waveguide, the center-of-mass of the mode is shifted more towards the outer sidewall. (b) Evaluation of the mode distribution along the red dashed cut line through the active region (AR). The ring waveguide supports several higher order radial modes.

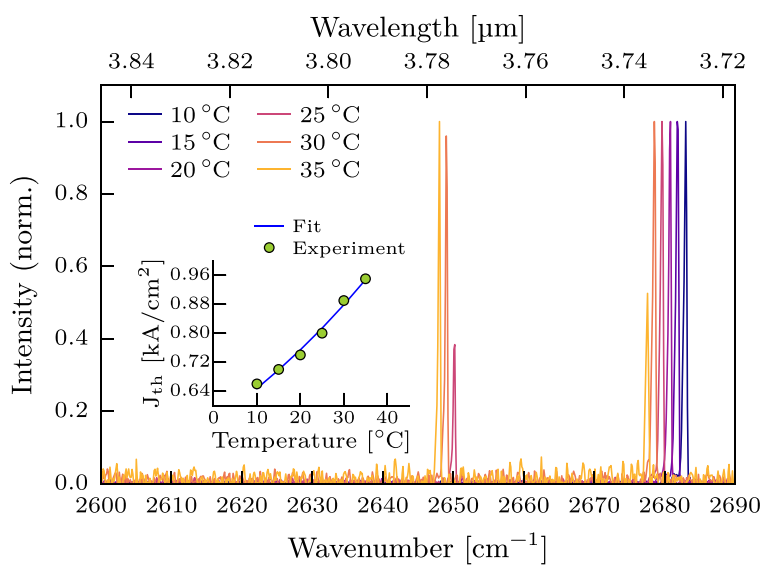

FIG. 4. Emission spectra from a substrate-emitting ring ICL at a drive current of $130 \mathrm{~mA}(80 \mathrm{kHz} / 30 \mathrm{~ns})$. As the heatsink temperature is increased above $20^{\circ} \mathrm{C}$, a second mode starts to appear at $\lambda \sim 3.77 \mu \mathrm{m}$. The inset shows the measured threshold current densities at different temperatures together with an exponential fit, from which a $J_{0}=0.55 \mathrm{kA} \mathrm{cm}^{-2}$ and a characteristic temperature of $T_{0}=66 \mathrm{~K}$ are extracted.

modes. As the waveguide width is reduced, also the losses for the higher order modes increase, while those for the fundamental mode remain low. A modal effective refractive index of $n_{\text {eff }}=3.42$ is calculated for the fundamental mode. From the measured emission wavelengths and the fabricated grating period, we find values of $n_{\text {eff }}=3.38$ for the peak at $\lambda=3.73 \mu \mathrm{m}$ and 3.418 for $\lambda=3.77 \mu \mathrm{m}$. These values are in good agreement with the results from the waveguide simulations. We conclude that the ring device lases in a higher order radial mode at temperatures below $25^{\circ} \mathrm{C}$. As the laser is operated at a low pulse duty-cycle, an increase in the temperature in the active region due to heat dissipation is negligible. Therefore, the shift of the emission wavelength can be related to a temperature-induced change of the refractive index. The shift of the emission peak around $3.73 \mu \mathrm{m}$ translates to a linear temperature tuning coefficient of $\Delta \lambda / \Delta T=0.3 \mathrm{~nm} /{ }^{\circ} \mathrm{C}$, as shown in Fig. 5. For a DFB ridge laser $(2.4 \mathrm{~mm} \times 9.8 \mu \mathrm{m})$ operating in continuous-wave, a comparable tuning coefficient of $\Delta \lambda / \Delta T=0.31 \mathrm{~nm} /{ }^{\circ} \mathrm{C}$ has been measured. ${ }^{12}$

Depending on the character of the optical transition from the conduction band to the valence band, the contributions of transverse magnetic (TM) or electric (TE) polarizations are

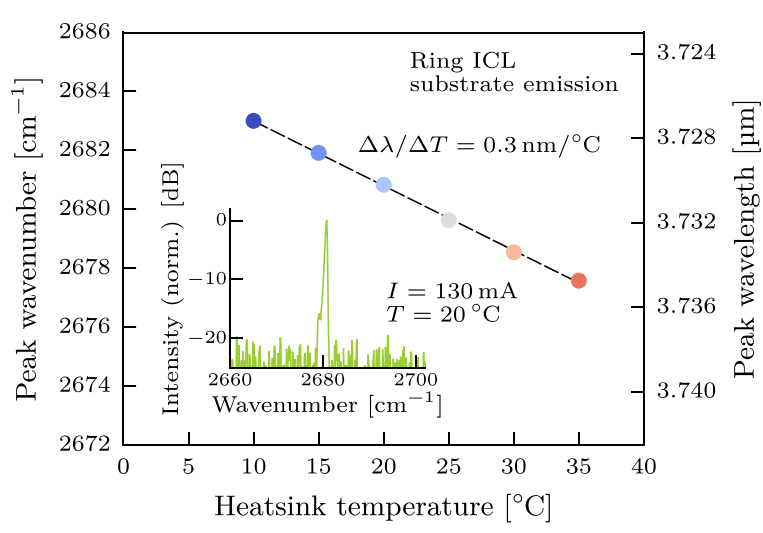

FIG. 5. Temperature tuning characteristics of a ring ICL device at a drive current of $130 \mathrm{~mA}(80 \mathrm{kHz} / 30 \mathrm{~ns})$. A linear temperature tuning coefficient of $0.3 \mathrm{~nm} /{ }^{\circ} \mathrm{C}$ is found. The inset shows the emission spectrum at $20^{\circ} \mathrm{C}$ on a semi-logarithmic scale with a SMSR $>15 \mathrm{~dB}$. 


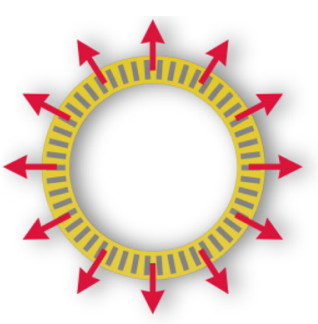

(a) Interband cascade laser

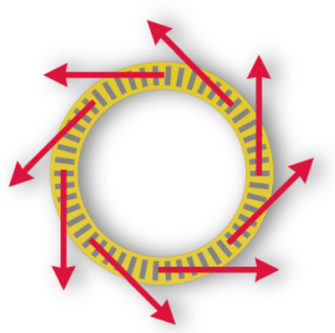

(b) Quantum cascade laser
FIG. 6. Schematic illustration of the obtained projected nearfield polarizations for ring lasers fabricated with two different device technologies. (a) For the ring ICL, a radial polarization of the electric field is found. (b) In contrast to that, an azimuthal orientation is observed for the ring QCL.

determined. The radiative recombination of an electron with a light-hole favors TM modes, while an electron to heavy-hole recombination enhances TE modes. The compressive strain in the GaInSb layer of typical ICL devices leads to a TE polarization of the light in the waveguide. ${ }^{29}$ In contrast to interband lasers, the intersubband selection rule restricts QCLs to favor TM polarized waveguide modes. As a consequence, the emitted light of ring ICLs is expected to have a different orientation of the electric field compared to its ring QCL counterpart. To verify this fundamental difference, projected nearfield images ${ }^{30}$ of these two device types are captured with a bolometer camera. Both devices have the same geometrical dimensions and emit light through the substrate. A polarizer is inserted in the beam path to examine the orientation of the electric field. Figure 6 shows an illustration of the observed nearfield polarizations in interband and intersubband ring lasers. For the ring ICL, the emission beam features a radial polarization. In contrast to that, the ring QCL shows an azimuthal orientation of the electric field, which has been also observed in previous studies. ${ }^{25,31}$ These experimental results underline the different nature of light generation in interband and intersubband cascade lasers, owing to the character of the optical transitions.

In conclusion, we have demonstrated ring interband cascade lasers with light emission at $\lambda \approx 3.7 \mu \mathrm{m}$ through the substrate. We fabricated a second-order DFB grating into the upper cladding layers and subsequently filled the etched grooves with metal. The measured output power and the slope efficiency are higher than those obtained with interband VCSEL devices ${ }^{14,15}$ but still considerably lower than from edge-emitting DFB ICLs. ${ }^{5}$ The ring ICL device is limited to pulsed operation due to poor heat dissipation capabilities. The hole in the submount is necessary to enable substrate emission, but it also prevents an efficient heat extraction in the vertical direction. Continuous-wave operation at room-temperature should be feasible with an appropriate mounting (e.g., epi-side down) of the device. Furthermore, we expect to increase the performance by improving the waveguide as well as the grating design. Measurements of the projected nearfield revealed a radial polarization for the ring ICL, whereas an azimuthal polarization is found for its intersubband counterpart.
The authors acknowledge the support by the Austrian Science Fund (FWF) Projects P26100-N27 (H2N) and NextLite (F4909-N23) and the State of Bavaria. H.D. acknowledges financial support through an APART fellowship from the Austrian Academy of Sciences.

${ }^{1}$ K. Vizbaras, A. Vizbaras, A. Andrejew, C. Grasse, S. Sprengel, and M.-C. Amann, Proc. SPIE 8277, 82771B (2012).

${ }^{2}$ J. Faist, F. Capasso, D. L. Sivco, C. Sirtori, A. L. Hutchinson, and A. Y. Cho, Science 264, 553 (1994).

${ }^{3}$ I. Vurgaftman, W. W. Bewley, C. L. Canedy, C. S. Kim, M. Kim, C. D. Merritt, J. Abell, and J. R. Meyer, IEEE J. Sel. Top. Quantum Electron. 19, 1200210 (2013).

${ }^{4}$ R. Q. Yang, Superlattices Microstruct. 17, 77 (1995).

${ }^{5}$ I. Vurgaftman, R. Weih, M. Kamp, J. R. Meyer, C. L. Canedy, C. S. Kim, M. Kim, W. W. Bewley, C. D. Merritt, J. Abell, and S. Höfling, J. Phys. D: Appl. Phys. 48, 123001 (2015).

${ }^{6}$ I. Vurgaftman, W. Bewley, C. Canedy, C. Kim, M. Kim, C. Merritt, J. Abell, J. Lindle, and J. Meyer, Nat. Commun. 2, 585 (2011).

${ }^{7}$ M. Kim, C. L. Canedy, W. W. Bewley, C. S. Kim, J. R. Lindle, J. Abell, I. Vurgaftman, and J. R. Meyer, Appl. Phys. Lett. 92, 191110 (2008).

${ }^{8}$ R. Weih, M. Kamp, and S. Höfling, Appl. Phys. Lett. 102, 231123 (2013).

${ }^{9}$ J. Scheuermann, R. Weih, M. von Edlinger, L. Nähle, M. Fischer, J. Koeth, M. Kamp, and S. Höfling, Appl. Phys. Lett. 106, 161103 (2015).

${ }^{10}$ C. S. Kim, M. Kim, W. W. Bewley, J. R. Lindle, C. L. Canedy, J. Abell, I. Vurgaftman, and J. R. Meyer, Appl. Phys. Lett. 95, 231103 (2009).

${ }^{11}$ C. S. Kim, M. Kim, J. Abell, W. W. Bewley, C. D. Merritt, C. L. Canedy, I. Vurgaftman, and J. R. Meyer, Appl. Phys. Lett. 101, 061104 (2012).

${ }^{12}$ R. Weih, L. Nähle, S. Höfling, J. Koeth, and M. Kamp, Appl. Phys. Lett. 105, 071111 (2014).

${ }^{13}$ M. von Edlinger, R. Weih, J. Scheuermann, L. Nähle, M. Fischer, J. Koeth, M. Kamp, and S. Höfling, Appl. Phys. Lett. 109, 201109 (2016).

${ }^{14}$ W. W. Bewley, C. L. Canedy, C. S. Kim, C. D. Merritt, M. V. Warren, I. Vurgaftman, J. R. Meyer, and M. Kim, Appl. Phys. Lett. 109, 151108 (2016).

${ }^{15}$ G. K. Veerabathran, S. Sprengel, A. Andrejew, and M.-C. Amann, Appl. Phys. Lett. 110, 071104 (2017).

${ }^{16}$ A. Lyakh, P. Zory, M. D'Souza, D. Botez, and D. Bour, Appl. Phys. Lett. 91, 181116 (2007).

${ }^{17}$ C. Schwarzer, E. Mujagić, S. I. Ahn, A. M. Andrews, W. Schrenk, W. Charles, C. Gmachl, and G. Strasser, Appl. Phys. Lett. 100, 191103 (2012).

${ }^{18}$ D. R. Scifres, R. D. Burnham, and W. Streifer, Appl. Phys. Lett. 28, 681 (1976).

${ }^{19}$ N. Matsumoto and K. Kumabe, Jpn. J. Appl. Phys., Part 1 16, 1395 (1977).

${ }^{20}$ J. P. Hohimer, D. C. Craft, G. R. Hadley, G. A. Vawter, and M. E. Warren, Appl. Phys. Lett. 59, 3360 (1991).

${ }^{21}$ E. Mujagić, L. K. Hoffmann, S. Schartner, M. Nobile, W. Schrenk, M. P. Semtsiv, M. Wienold, W. T. Masselink, and G. Strasser, Appl. Phys. Lett. 93, 161101 (2008).

${ }^{22}$ R. Noll and S. Macomber, IEEE J. Quantum Electron. 26, 456 (1990).

${ }^{23}$ C. Sigler, J. D. Kirch, T. Earles, L. J. Mawst, Z. Yu, and D. Botez, Appl. Phys. Lett. 104, 131108 (2014).

${ }^{24}$ R. Szedlak, C. Schwarzer, T. Zederbauer, H. Detz, A. Maxwell Andrews, W. Schrenk, and G. Strasser, Appl. Phys. Lett. 104, 151105 (2014).

${ }^{25}$ C. Schwarzer, R. Szedlak, S. Il Ahn, T. Zederbauer, H. Detz, A. Maxwell Andrews, W. Schrenk, and G. Strasser, Appl. Phys. Lett. 103, 081101 (2013).

${ }^{26}$ N. Finger, W. Schrenk, and E. Gornik, IEEE J. Quantum Electron. 36, 780 (2000).

${ }^{27}$ H. Kogelnik and C. V. Shank, J. Appl. Phys. 43, 2327 (1972).

${ }^{28}$ J.-W. Shi, C.-H. Jiang, K.-M. Chen, J.-L. Yen, and Y.-J. Yang, Appl. Phys. Lett. 87, 031109 (2005).

${ }^{29}$ K. Ryczko and G. Sęk, AIP Adv. 6, 115020 (2016).

${ }^{30}$ J. Lin, J. K. Gamelin, G. T. Du, S. Wang, M. Hong, and J. P. Mannaerts, Appl. Phys. Lett. 60, 2851 (1992).

${ }^{31}$ Y. Bai, S. Tsao, N. Bandyopadhyay, S. Slivken, Q. Y. Lu, D. Caffey, M. Pushkarsky, T. Day, and M. Razeghi, Appl. Phys. Lett. 99, 261104 (2011). 